\title{
Optimal Investment Behavior for Regret-Averse Competitive Firms under Price Uncertainty
}

\author{
Jinwu Huang ${ }^{1}$ \\ ${ }^{1}$ School of Finance, Shanghai University of Finance and Economics, Shanghai, China \\ Correspondence: Jinwu Huang, School of Finance, Shanghai University of Finance and Economics, 200433 \\ Shanghai, China. E-mail: eyesonme_hmily@msn.com
}

Received: May 31, 2013

doi:10.5539/ijef.v5n8p109
Accepted: June 25, $2013 \quad$ Online Published: July 26, 2013

URL: http://dx.doi.org/10.5539/ijef.v5n8p109

\begin{abstract}
In this paper, we try to analyze the optimal capital and labor input of a competitive firm under price uncertainty from the perspective of regret aversion rather than of risk aversion. We show that the optimal input of capital and labor for the competitive firm under certain price are higher than those for the regret-averse competitive firm under price uncertainty. Moreover, we prove that the optimal input will increase or decrease with the movement of the regret factor. Besides, we find that the higher the expected probability of the output price turns out to be high and sales turn to be good, the less impacts of the changes of the weight of regret aversion relative to risk aversion would on the optimal capital and labor input.
\end{abstract}

Keywords: competitive firm, optimal input, price uncertainty, regret aversion

\section{Introduction}

Decision making under uncertainty has been appealing to many scholars in many fields such as general equilibrium theory, monetary economics, public economics, investment theory and so on. Regarding these applications, one can read Sandmo (1971), Leland (1972) and etc at length. Within these papers, the authors assume that the preferences of a firm are defined only on the foundation of profits, and that a Von Neumann-Morgenstern (Hereafter VNM ) type utility functions can be used to describe these preferences. As we all know, utility theory has been proved to be adequate for describing the behavior of decision makers. Nevertheless, there are some cases in the real world, in which the VNM type utility theory yields poor results due to the uni-criterion assumption. In view of this point, Landsberger and Subotnik (1976) attempted to define the objective of a firm by a utility function based on firm's profits and some other factors. They just studied, however, the decision making process without uncertainty in their paper.

In this paper, we will apply a bi-criterion utility function to express the objective of a competitive firm, and will focus on the firm's behavior under uncertainty of output price. To this end, we introduce regret, which implies that a decision maker may seek to minimize it that he will feel if it would turn out that he has made the wrong decision, as another factor into the utility function of the firm in addition to the profit. Of course, it's sensible to consider regret in practice not only because decision makers indeed take regret into account in decision making process, but also because regret consideration can explain some interesting economic phenomena. Many researchers have analyzed this criterion in the past, and it has been argued that regret is important in determining the behavior of junior executives and is a reasonable criterion to statisticians. Additionally, it has been suggested that regret is viewed as a possible explanation for Allais' paradox and is the only criterion to make a hedging strategy optimal. Regarding the issues expressed above, we can refer to Marschak (1974), Paroush and Venezia (1979), and so on.

Generally, we assume the VNM type utility functions are risk-averse, but some researches offered evidence that violates the conventional expected utility theory with risk aversion. By contrary, they proposed there were alternative choices for utility functions other than risk aversion. Of which, Paroush and Venezia is the first to apply a regret-averse model to the competitive firm by considering the firm with a regret utility function, Loomes and Sugden (1982) showed that regret aversion is an alternative theory and is a rational choice under uncertainty. In particular, Loomes and Sugden, and Bell (1983) presented a formal analysis of regret theory. In recent years, considerable literatures studied firm's behavior by supposing firms are regret-averse instead of supposing firms are risk-averse. Notice that they define regret in their papers as the disutility of not selecting the 
ex-post optimal alternative. That is, firms might regret producing less if the output price turns out to be very high and sales turn out to be very good, and firms might regret producing more if the output price turns out to be low and sales turn out to be poor. Again, we recall that Paroush and Venezia derived the conditions under which the optimal output in certain framework is higher than that under uncertainty in an equivalent case, although the shortage of the paper is that their results rely on the relative importance of the regret term and the firm's profits. Luckily, with the development of research, more specific and tractable regret-averse functions than that presented by Paroush and Vnezia have been proposed in many works such as in Braun and Muermann (2004), Muermann et al. (2006), Mulandzi et al. (2008) and so forth. Specifically, using such type of more specific and tractable regret-averse functions, Braun and Muermann (2004) examined optimal insurance purchase decisions of individuals and found that individuals with regret-theoretical preferences adjust away from the extremes of full insurance and no insurance coverage. This prediction not only holds for both coinsurance and deductible contracts, but also can explain the frequently observed preferences for low deductibles in markets for personal insurance. Muermann et al. (2006) studied the portfolio's optimal allocation problem in a defined contribution pension plan for a regret-averse investor, who confronts with a risky and a risk-free asset at the same time. The results demonstrate that anticipated disutility from regret has potent effects on investment choices. Relative to investors with risk aversion, investors who take regret into account will hold more stock when the equity premium is low but less stock when the equity premium is high. Moreover, regret increases the regret-averse investor's willingness to pay for a guarantee when the portfolio is relatively risky, but decreases it when the portfolio is relatively safe. Mulandzi et al. (2008) analyzed the optimal allocation between loans and treasuries for a regret-averse bank and investigated the investment of bank funds in loans and Treasuries with the aim of generating an optimal final fund level. Taking risk and regret into account in the utility function and applying optimization theory, they provide a comparison between risk- and regret-averse banks in terms of optimal asset allocation between loans and Treasuries. Furthermore, they comment on the claim that an investment away from loans towards Treasuries is responsible for credit crunches in the banking industry. As to more references, one can refer to Stoltz and Lugosi (2005), Michenaud and Solink (2008), Hayashi (2009), Ma and Xu (2009), Petersen (2010), Renou and Schlag (2010), Stoye (2011), Sheng (2012), Tsai (2012), Wong (2011, 2012) and some references therein.

By adopting the more specific and tractable regret-averse function, we discuss the properties of firm's behavior when optimal input is invested for the regret-averse competitive firm under uncertain price of output. What's more important, our paper makes a few slight contributions to existing literatures. Above all, our paper is the first to apply the two-attribute utility function to study the investment behavior of competitive firms with regret aversion as well as risk aversion under price uncertainty. Then, our model enables different firms to possess different regrets if their utility functions on regret terms are different, which circumvent the limitation that utility functions of regret-averse firms with different regret terms possesses the same regret. The last but not the least, our framework allows us to make comparative statics of the optimal input by changing the parameter of the regret term. By the way, our setup not only represents the production theory for competitive firms with risk aversion, but also represents the production theory for competitive firms with regret aversion.

The remaining of this paper is organized as follows. In Section 2, we introduce the preliminaries for our model in detail. Applying a more specific and tractable regret-averse function, Section 3 analyzes the behavior of competitive firm with regret aversion under price uncertainty. An illustration for our model is shown in Section 4 . Finally, Section 5 presents the conclusions.

\section{Preliminaries}

In this paper, we will introduce the decision making process elaborately of a regret-averse competitive firm under uncertainty of output price. For our purpose, we present the hypotheses explicitly as below.

(H1) The competitive firm's production function is defined as

$$
Q=F(K, L)
$$

where, $Q$ is the quantity of the products, $K$ and $L$ are respectively capital and labor input, function $F$ is continuous, twice differentiable, and strictly quasi-concave, i.e., $F_{K K}, F_{L L}<0$, and $F_{K K} F_{L L} \geq F_{K L}^{2}$ with $F_{K L}>0$. Certainly, the production function $F$ is increasing with respect to $K$ and $L$, i.e., $F_{K}, F_{L}>0$.

(H2) The competitive firm sells its output at an uncertain price $p$, which follows

$$
p=\hat{p}+\sigma \varepsilon,
$$

$\hat{p}$ is the expected price, i.e., $\hat{p}=E(p), \sigma$ is the standard deviation of price $p$, and $\varepsilon$ is a random variable that satisfies 


$$
E(\varepsilon)=0, \quad \operatorname{Var}(\varepsilon)=1, \quad \operatorname{Prob}(\varepsilon>-\hat{p} / \sigma)=1 .
$$

(H3) We define the two-attribute utility function $u(\pi, R)$ of the regret-averse competitive firm under price uncertainty by

$$
u(\pi, R)=v(\pi)-\lambda g(R)
$$

Herein, $v$ is a utility function such that $v^{\prime}>0, v^{\prime}<0$ to reflect the risk aversion that accounts for the first attribute, while the second attribute that reflects regret aversion is explained by an increasing and strictly convex function $g$ with $g(0)=0$ and $g^{\prime}>0, g^{\prime \prime}>0$ on interval $(0,+\infty), \lambda$ is a nonnegative parameter to measure the weight of regret-averse attribute relative to risk-averse attribute. Notice that the two-attribute utility function $u(\pi, R)$ will collapse to the conventional VNM type utility function when $\lambda=0$, and then the firm would be a maximizer of expected utility under risk aversion only.

(H4) The firm's profit function $\pi$ and the regret function $R$ are denoted respectively by

$$
\begin{gathered}
\pi=p Q-r K-w L \\
R=v\left(\pi^{\max }\right)-v(\pi)
\end{gathered}
$$

where, $w$ in expression (3) represents the wage rate and $r$ therein represents the capital rent, $\pi^{\max }$ in formula (4) is the ex-post optimal profit without price uncertainty. Moreover, the value of the regret term $R$ is nonnegative since $v$ is increasing. Notice that we dropped the fixed cost in the firm's profit function, because doing this will not make any influnce to our results.

Obviously, from hypothesis (H3), there holds $u_{\pi}>0, u_{R}<0$, which means the firm likes profit but dislikes regret. And furthermore, $u_{\pi \pi}<0, u_{R R}<0$, which implies the utility function $u(\pi, R)$ is concave on the profit $\pi$ and the regret $R$ to reflect risk and regret aversion, respectively.

At the end, according to the definitions of $u(\pi, R)$ and $R$ that shown in expressions (2) and (4), we claim that $u(\pi$, $R)$ is a function of $\pi$ in essence because $\pi^{\max }$ is the ex-post optimal profit under certain price. Hence, we can rewrite the two-attribute regret-averse utility function as

$$
u(\pi)=v(\pi)-\lambda g\left(v\left(\pi^{\max }\right)-v(\pi)\right)
$$

which suggests the pleasant experience of possessing the profit $\pi$ depends not only on $v(\pi)$ but also on regret term $R=v\left(\pi^{\max }\right)-v(\pi)$, i.e., the difference between the value the firm assigns to the ex-post optimal profit level $\pi^{\max }$ that could have achieved and the value it assigns to its actual final level of profit $\pi$. Exactly speaking, possess the maximal profit $\pi^{\max }$ is better off possessing profit $\pi$ and suffering regret experience as well. To this sense, the attribute of regret aversion depicted by function $g$ indicates that the more pleasurable the consequence might have been, the more regret will be undergone.

\section{Behavior of Competitive Firms with Regret Aversion}

To begin, we declare that the objective of the competitive firm with regret aversion under price uncertainty is to maximize the expectation of $u(\pi)$ by selecting the capital and labor input. Mathematically, we can write our problem as

$$
\max _{K, L} E u(\pi)
$$

Here, $E$ is the linear expectation operator, $u(\pi)$ is defined as in expression (5), and $\pi$ is subject to constraint (3). It's clear that the objective proposed for regret-averse competitive firm under uncertainty here is different from the one for risk-averse competitive firm under uncertainty, since the latter's objective is to maximize the expectation of utility function only for the profit, but the former's objective that shown above is to maximize the expectation of utility function on the profit and regret as well.

There are many merits to take advantage of such a model setting. For one thing, both the theory for competitive firms with risk aversion $(\lambda=0)$ and the theory for competitive firms with regret aversion $(\lambda>0)$ are involved. For another thing, we use $v\left(\pi^{\max }\right)-v(\pi)$ rather than $\pi^{\max }-\pi$ to index the regret term in the model setting, which enables different firms to own different regret as long as their utility functions on the regret term are different, and circumvents the shortage that different firms possess the same regret though their utility functions on regret are different. Besides, we can see that the bigger the value of $\lambda$, the stronger the attitude of regret. And so on.

Subsequently, we will adopt the more specific and tractable regret-averse utility function $u(\pi)$ to investigate the optimal behavior of competitive firms with regret aversion under price uncertainty.

For our purpose, differentiating $E u(\pi)$ with respect to $K$ and $L$, respectively, and taking equalities (3) and (5) into account, we see the first-order conditions are 


$$
\begin{aligned}
& \frac{\partial E u(\pi)}{\partial K}=E\left\{u^{\prime}(\pi)\left(p F_{K}-r\right)\right\}, \\
& \frac{\partial E u(\pi)}{\partial L}=E\left\{u^{\prime}(\pi)\left(p F_{L}-w\right)\right\},
\end{aligned}
$$

and the second-order conditions are

$$
\begin{aligned}
& \frac{\partial^{2} E u}{\partial K^{2}}=E\left\{u^{\prime \prime}(\pi)\left(p F_{K}-r\right)^{2}+u^{\prime}(\pi) p F_{K K}\right\}, \\
& \frac{\partial^{2} E u}{\partial L^{2}}=E\left\{u^{\prime \prime}(\pi)\left(p F_{L}-r\right)^{2}+u^{\prime}(\pi) p F_{L L}\right\},
\end{aligned}
$$

where, $u^{\prime}(\pi)=v^{\prime}(\pi)-\lambda g^{\prime}\left(v\left(\pi^{\max }\right)-v(\pi)\right) v^{\prime}(\pi)$, while $u{ }^{\prime \prime}(\pi)=v^{\prime \prime}-\lambda g^{\prime \prime} v^{\prime 2}+\lambda g{ }^{\prime \prime} v^{\prime \prime}$.

Reminding that $F_{K K}, F_{L L}<0, v^{\prime}>0, v^{\prime \prime}<0 \quad$ and $g^{\prime}, g^{\prime \prime}>0, \lambda \geq 0$, it's evident to see $\partial^{2} E u / \partial K^{2}, \partial^{2} E u / \partial L^{2}<0$. Therefore, we can claim that $K^{*}$ and $L^{*}$ are the optimal capital and labor input for the maximal expected utility of the competitive firm with regret aversion under price uncertainty, provided that there holds $\partial E u / \partial K=0$ at $K=K^{*}$ and $\partial E u / \partial L=0$ at $L=L^{*}$. As a matter of fact, we have the following Lemma.

Lemma. Let $K^{*}$ and $L^{*}$ be the optimal capital and labor input chosen to maximize the objective of the regret-averse competitive firm with uncertain price that defined in the optimal problem (6), then under the hypotheses (H1)- (H4), we see

$$
\begin{array}{ll}
F_{K}\left(K^{*}, L^{*}\right)<\frac{r}{E p}, & F_{L}\left(K^{*}, L^{*}\right)<\frac{w}{E p}, \quad \text { if } \operatorname{Cov}\left(u^{\prime}(\pi), p\right)>0, \\
F_{K}\left(K^{*}, L^{*}\right)>\frac{r}{E p}, & F_{L}\left(K^{*}, L^{*}\right)>\frac{w}{E p}, \quad \text { if } \operatorname{Cov}\left(u^{\prime}(\pi), p\right)<0, \\
F_{K}\left(K^{*}, L^{*}\right)=\frac{r}{E p}, & F_{L}\left(K^{*}, L^{*}\right)=\frac{w}{E p}, \quad \text { if } \operatorname{Cov}\left(u^{\prime}(\pi), p\right)=0 .
\end{array}
$$

Put differently, if $u^{\prime}(\pi)$ is increasing with $P$, then $F_{K}\left(K^{*}, L^{*}\right)<\frac{r}{E p}, F_{L}\left(K^{*}, L^{*}\right)<\frac{w}{E p}$; if $u^{\prime}(\pi)$ is decreasing with $P$, then $F_{K}\left(K^{*}, L^{*}\right)>\frac{r}{E p}, F_{L}\left(K^{*}, L^{*}\right)>\frac{w}{E p} ;$ and if $u^{\prime}(\pi)$ is uncorrelated with $P$, then $F_{K}\left(K^{*}, L^{*}\right)=\frac{r}{E p}, F_{L}\left(K^{*}, L^{*}\right)=\frac{w}{E p}$, which is equivalent to the case without price uncertainty.

Proof. Actually, by simple computation, it's easy to rewrite equations (7)-(8) as

$$
\begin{gathered}
\frac{\partial E u}{\partial K}=F_{K} \operatorname{Cov}\left(u^{\prime}(\pi), p\right)+\left(\hat{p} F_{K}-r\right) E u^{\prime}(\pi), \\
\frac{\partial E u}{\partial L}=F_{L} \operatorname{Cov}\left(u^{\prime}(\pi), p\right)+\left(\hat{p} F_{L}-w\right) E u^{\prime}(\pi) .
\end{gathered}
$$

Thus, by setting $\partial E u / \partial K=0, \partial E u / \partial L=0$, and considering $F_{K}, F_{L}>0$ as well as $u^{\prime}(\pi)>0, \hat{p}>0$, the results shown in the Lemma is obtained. The proof is completed.

We remark that $F_{K}\left(K^{*}, L^{*}\right)=\frac{r}{E p}, \quad F_{L}\left(K^{*}, L^{*}\right)=\frac{w}{E p}$ just hold under the price without uncertainty, i.e., $p=\hat{p}$. While under price with uncertainty, $F_{K}\left(K^{*}, L^{*}\right)<\frac{r}{E p}, \quad F_{L}\left(K^{*}, L^{*}\right)<\frac{w}{E p} \quad$ will hardly happen in reality. To elaborate the reasons in detail, we present the Theorem 1 below. 
Theorem 1. Let $K^{*}$ and $L^{*}$ be the optimal capital and labor input chosen to maximize the objective of the regret-averse competitive firm with uncertain price that defined in optimal problem (6), then under the hypotheses (H1)- (H4), there must hold $F_{K}\left(K^{*}, L^{*}\right)>\frac{r}{E p}, \quad F_{L}\left(K^{*}, L^{*}\right)>\frac{w}{E p}$, which implies that both the optimal input of capital and labor under price uncertainty are less than those under certain price.

Proof. Letting $\partial E u / \partial K=0$, then by equality (7), it yields

$$
E\left\{u^{\prime}(\pi)(p-\hat{p})\right\}=E\left\{u^{\prime}(\pi)\left(r / F_{K}-\hat{p}\right)\right\} .
$$

Taking equations (1) and (3) into account, we have

$$
\pi-E \pi=(p-\hat{p}) Q .
$$

Reminding that $u(\pi)$ is a concave function, so following formula (12) above, we obtain

$$
\begin{cases}u^{\prime}(\pi) \leq u^{\prime}(E \pi), & p \geq \hat{p}, \\ u^{\prime}(\pi)>u^{\prime}(E \pi), & p<\hat{p} .\end{cases}
$$

Directly, we can get

$$
u^{\prime}(\pi)(p-\hat{p}) \leq u^{\prime}(E \pi)(p-\hat{p})
$$

Taking expectation on both sides of inequality (13), and noting that $u^{\prime}(E \pi)$ is a given value, there holds

$$
E\left\{u^{\prime}(\pi)(p-\hat{p}\} \leq u^{\prime}(E \pi) E(p-\hat{p})=0 .\right.
$$

Observing formulae (11) and (14), we can see

$$
E\left\{u^{\prime}(\pi)\left(r / F_{K}-\hat{p}\right)\right\} \leq 0 .
$$

And then, we have

$$
F_{K}\left(K^{*}, L^{*}\right) \geq r / \hat{p},
$$

because $u^{\prime}(\pi)>0, r / F_{K}-\hat{p}$ is a determined number.

Repeating the same argument, we can obtain

$$
F_{L}\left(K^{*}, L^{*}\right) \geq w / \hat{p} .
$$

Especially, we should notice that $F_{K}\left(K^{*}, L^{*}\right)=r / \hat{p}, F_{L}\left(K^{*}, L^{*}\right)=w / \hat{p}$ hold just under the condition $p=\hat{p}=E p$, i.e., $p$ is a constant variable. In other words, under price uncertainty, there only holds $F_{K}\left(K^{*}, L^{*}\right)>r / \hat{p}, F_{L}\left(K^{*}, L^{*}\right)>w / \hat{p}$, which means that both the optimal input of capital and labor under price uncertainty are less than those under certain price since $F_{K}$ and $F_{L}$ are decreasing functions with respect to $K$ and $L$, respectively. The proof is completed.

Finally, we continue to examine the question that how the optimal behavior changes with the movement of the regret factor. To this end, Theorem 2 is proposed straightforwardly.

Theorem 2. Let $K^{*}$ and $L^{*}$ be the optimal capital and labor input chosen to maximize the objective of the regret-averse competitive firm with uncertain price that defined in optimal problem (6), then under the hypotheses (H1)- (H4), there yields

$$
\begin{aligned}
& \partial K^{*} / \partial \lambda>0, \quad \text { if }\left(\hat{p} F_{K}-r\right) / F_{K}>\operatorname{Cov}\left(u^{\prime}(\pi)-v^{\prime}(\pi),-p\right) / E\left\{u^{\prime}(\pi)-v^{\prime}(\pi)\right\}, \\
& \partial K^{*} / \partial \lambda<0, \quad \text { if }\left(\hat{p} F_{K}-r\right) / F_{K}<\operatorname{Cov}\left(u^{\prime}(\pi)-v^{\prime}(\pi),-p\right) / E\left\{u^{\prime}(\pi)-v^{\prime}(\pi)\right\} ; \\
& \partial L^{*} / \partial \lambda>0, \quad \text { if }\left(\hat{p} F_{L}-w\right) / F_{K}>\operatorname{Cov}\left(u^{\prime}(\pi)-v^{\prime}(\pi),-p\right) / E\left\{u^{\prime}(\pi)-v^{\prime}(\pi)\right\}, \\
& \partial L^{*} / \partial \lambda<0, \quad \text { if }\left(\hat{p} F_{L}-w\right) / F_{L}<\operatorname{Cov}\left(u^{\prime}(\pi)-v^{\prime}(\pi),-p\right) / E\left\{u^{\prime}(\pi)-v^{\prime}(\pi)\right\} .
\end{aligned}
$$

Particularly, neither the sign of $\partial K^{*} / \partial \lambda$ nor of $\partial L^{*} / \partial \lambda$ can be determined definitely if 
$\left(\hat{p} F_{K}-r\right) / F_{K}=\operatorname{Cov}\left(u^{\prime}(\pi)-v^{\prime}(\pi),-p\right) / E\left\{u^{\prime}(\pi)-v^{\prime}(\pi)\right\}$

and

$\left(\hat{p} F_{L}-w\right) / F_{L}=\operatorname{Cov}\left(u^{\prime}(\pi)-v^{\prime}(\pi),-p\right) / E\left\{u^{\prime}(\pi)-v^{\prime}(\pi)\right\}$.

Proof. Since $\partial^{2} E u / \partial K^{2}<0, \partial^{2} E u / \partial L^{2}<0$, thus, the optimal capital input $K^{*}$ and labor input $L^{*}$ can be obtained by solving equations $\partial E u / \partial K=0, \partial E u / \partial L=0$. By equations (7)-(8), we see that $K^{*}, L^{*}$ satisfy

$$
\begin{aligned}
& E\left\{u^{\prime}(\pi)\left(p F_{K}-r\right)\right\}=0, \\
& E\left\{u^{\prime}(\pi)\left(p F_{L}-w\right)\right\}=0 .
\end{aligned}
$$

Naturally, $K^{*}, L^{*}$ are the solutions for equations

$$
\begin{gathered}
E\left\{\left(v^{\prime}(\pi)+\lambda g^{\prime} v^{\prime}(\pi)\right)\left(p F_{K}-r\right)\right\}=0, \\
E\left\{\left(v^{\prime}(\pi)+\lambda g^{\prime} v^{\prime}(\pi)\right)\left(p F_{L}-w\right)\right\}=0,
\end{gathered}
$$

because $u^{\prime}(\pi)=v^{\prime}(\pi)+\lambda g^{\prime}\left(v\left(\pi^{\mathrm{max}}\right)-v(\pi)\right) v^{\prime}(\pi)$. Evidently, the two equations indicate $\lambda$ can be expressed by $K^{*}, L^{*}$. That is, $\lambda$ is a function of $K^{*}, L^{*}$.

We next examine the dynamic relationships between $K^{*}$ and $\lambda, L^{*}$ and $\lambda$.

Differentiating both sides of equation (15) with respect to $K^{*}$ and equation (16) with respect to $L^{*}$, we have

$$
\begin{aligned}
& \frac{\partial \lambda}{\partial K^{*}} \cdot E\left\{g^{\prime} v^{\prime}\left(p F_{K}-r\right)\right\}=-E\left\{\left(v^{\prime \prime}+\lambda\left(g^{\prime} v^{\prime \prime}-g^{\prime \prime} v^{\prime 2}\right)\right)\left(p F_{K}-r\right)^{2}\right\}-E\left\{\left(v^{\prime}+\lambda g^{\prime} v^{\prime}\right) p F_{K K}\right\}, \\
& \frac{\partial \lambda}{\partial L^{*}} \cdot E\left\{g^{\prime} v^{\prime}\left(p F_{L}-w\right)\right\}=-E\left\{\left(v^{\prime \prime}+\lambda\left(g^{\prime} v^{\prime \prime}-g^{\prime \prime} v^{\prime 2}\right)\right)\left(p F_{L}-w\right)^{2}\right\}-E\left\{\left(v^{\prime}+\lambda g^{\prime} v^{\prime}\right) p F_{L L}\right\} .
\end{aligned}
$$

Applying $u^{\prime}=v^{\prime}+\lambda g^{\prime} v^{\prime}$, and $u^{\prime \prime}=v^{\prime \prime}+\lambda\left(g^{\prime} v^{\prime \prime}-g^{\prime \prime} v^{\prime 2}\right)$, we get

$$
\begin{aligned}
& \frac{\partial \lambda}{\partial K^{*}} \cdot E\left\{g^{\prime} v^{\prime}\left(p F_{K}-r\right)\right\}=-E\left\{u^{\prime \prime}\left(p F_{K}-r\right)^{2}\right\}-E\left\{u^{\prime} p F_{K K}\right\}>0, \\
& \frac{\partial \lambda}{\partial L^{*}} \cdot E\left\{g^{\prime} v^{\prime}\left(p F_{L}-w\right)\right\}=-E\left\{u^{\prime \prime}\left(p F_{L}-w\right)^{2}\right\}-E\left\{u^{\prime} p F_{L L}\right\}>0,
\end{aligned}
$$

since $u^{\prime}>0, u^{\prime \prime}<0, F_{K K}, F_{L L}<0$ and $p>0$.

To judge the signs of $\partial \lambda / \partial K^{*}, \partial \lambda / \partial L^{*}$ definitely, we only need to judge the signs of $E\left\{g^{\prime} v^{\prime}\left(p F_{K}-r\right)\right\}, E\left\{g^{\prime} v^{\prime}\left(p F_{L}-w\right)\right\}$, respectively. Actually, we know

$$
\begin{aligned}
E\left\{g^{\prime} v^{\prime}\left(p F_{K}-r\right)\right\} & =\operatorname{Cov}\left(g^{\prime} v^{\prime}, p F_{K}-r\right)+E\left\{g^{\prime} v^{\prime}\right\}\left(\hat{p} F_{K}-r\right) \\
& =F_{K} \operatorname{Cov}\left(g^{\prime} v^{\prime}, p\right)+E\left\{g^{\prime} v^{\prime}\right\}\left(\hat{p} F_{K}-r\right) \\
& =\frac{F_{K}}{\lambda} \operatorname{Cov}\left(u^{\prime}-v^{\prime}, p\right)+\frac{1}{\lambda} E\left\{u^{\prime}-v^{\prime}\right\}\left(\hat{p} F_{K}-r\right) .
\end{aligned}
$$

Similarly, we obtain

$$
E\left\{g^{\prime} v^{\prime}\left(p F_{L}-w\right)\right\}=\frac{F_{L}}{\lambda} \operatorname{Cov}\left(u^{\prime}-v^{\prime}, p\right)+\frac{1}{\lambda} E\left\{u^{\prime}-v^{\prime}\right\}\left(\hat{p} F_{L}-w\right) .
$$

At this point, we see 


$$
\begin{aligned}
& \partial \lambda / \partial K^{*}>0, \quad \text { if }\left(\hat{p} F_{K}-r\right) / F_{K}>\operatorname{Cov}\left(u^{\prime}-v^{\prime},-p\right) / E\left\{u^{\prime}-v^{\prime}\right\}, \\
& \partial \lambda / \partial K^{*}<0, \quad \text { if }\left(\hat{p} F_{K}-r\right) / F_{K}<\operatorname{Cov}\left(u^{\prime}-v^{\prime},-p\right) / E\left\{u^{\prime}-v^{\prime}\right\}, \\
& \partial \lambda / \partial L^{*}>0, \quad \text { if }\left(\hat{p} F_{L}-w\right) / F_{L}>\operatorname{Cov}\left(u^{\prime}-v^{\prime},-p\right) / E\left\{u^{\prime}-v^{\prime}\right\}, \\
& \partial \lambda / \partial L^{*}<0, \quad \text { if }\left(\hat{p} F_{L}-w\right) / F_{L}<\operatorname{Cov}\left(u^{\prime}-v^{\prime},-p\right) / E\left\{u^{\prime}-v^{\prime}\right\} .
\end{aligned}
$$

In particular, the signs of $\partial \lambda / \partial K^{*}, \partial \lambda / \partial L^{*}$ are undetermined if $\left(\hat{p} F_{K}-r\right) / F_{K}=\operatorname{Cov}\left(u^{\prime}-v^{\prime},-p\right) / E\left\{u^{\prime}-v^{\prime}\right\},\left(\hat{p} F_{L}-r\right) / F_{L}=\operatorname{Cov}\left(u^{\prime}-v^{\prime},-p\right) / E\left\{u^{\prime}-v^{\prime}\right\} \quad$ The proof is completed.

\section{Illustration}

In this section, we illustrate the findings we obtained in Theorems 1-2 in Section 3.

For simplicity and operability, the production function $F(K, L)$ of the regret-averse competitive firm is adopted by Cobb-Douglas type function, namely

$$
F(K, L)=A K^{\alpha} L^{\beta}, \quad 0<\alpha, \beta<1, \quad A>0 .
$$

It's clear to see that $F(K, L)=A K^{\alpha} L^{\beta}$ satisfies the quasi-concave conditions. Without loss of generality, we set $\alpha=1 / 2, \beta=1 / 4$ and normalize the technology $A$ to unit one hereafter, i.e., $A=1$. Secondly, we assume function $v(x)=x^{1 / 2}$ and function $g(x)=x^{6 / 5}$, which satisfy the hypothesis (H3). Lastly, we suppose the price $p=4$ with expected probability $q$ or $p=8$ with expected probability $1-q$, and $w=1 / 4, r=1 / 2$.

Under the theory developed in this paper, the competitive firm with regret aversion under price uncertainty will choose optimal input by maximizing the expected utility $E u(\pi)$, that is

$$
\begin{aligned}
& \max _{K, L} E u(\pi)=\max _{K, L}\left\{q\left(\sqrt{4 K^{1 / 2} L^{1 / 4}-K / 2-L / 4}-\lambda\left(8-\sqrt{4 K^{1 / 2} L^{1 / 4}-K / 2-L / 4}\right)^{6 / 5}\right)\right. \\
& \left.+(1-q)\left(\sqrt{8 K^{1 / 2} L^{1 / 4}-K / 2-L / 4}-\lambda\left(32-\sqrt{8 K^{1 / 2} L^{1 / 4}-K / 2-L / 4}\right)^{6 / 5}\right)\right\} .
\end{aligned}
$$

Setting $\partial E u(\pi) / \partial K=0, \partial E u(\pi) / \partial L=0$, we have

$$
\begin{aligned}
& \frac{1-q}{\sqrt{B_{1}}}\left(4 K^{-\frac{1}{2}} L^{\frac{1}{4}}-\frac{1}{2}\right)\left(\frac{1}{2}+\frac{3 \lambda}{5}\left(32-\sqrt{B_{1}}\right)^{\frac{1}{5}}\right)=\frac{q}{\sqrt{B_{2}}}\left(\frac{1}{2}-2 K^{-\frac{1}{2}} L^{\frac{1}{4}}\right)\left(\frac{1}{2}+\frac{3 \lambda}{5}\left(8-\sqrt{B_{2}}\right)^{\frac{1}{5}}\right), \\
& \frac{1-q}{\sqrt{B_{1}}}\left(2 K^{\frac{1}{2}} L^{-\frac{3}{4}}-\frac{1}{4}\right)\left(\frac{1}{2}+\frac{3 \lambda}{5}\left(32-\sqrt{B_{1}}\right)^{\frac{1}{5}}\right)=\frac{q}{\sqrt{B_{2}}}\left(\frac{1}{4}-K^{\frac{1}{2}} L^{-\frac{3}{4}}\right)\left(\frac{1}{2}+\frac{3 \lambda}{5}\left(8-\sqrt{B_{2}}\right)^{\frac{1}{5}}\right),
\end{aligned}
$$

where, $B_{1}=8 K^{1 / 2} L^{1 / 4}-K / 2-L / 4, B_{2}=4 K^{1 / 2} L^{1 / 4}-K / 2-L / 4$, then we get $K^{*}=L^{*}$.

Under price uncertainty, it's easy to obtain

Case I. The expected probability of $p=4$ is $q=1 / 2$ 


$$
\left\{\begin{array}{l}
K^{*}=L^{*}=565.683, \text { if } \lambda=0 \\
K^{*}=L^{*}=582.887, \text { if } \lambda=0.25 \\
K^{*}=L^{*}=591.496, \text { if } \lambda=0.5 \\
K^{*}=L^{*}=596.643, \text { if } \lambda=0.75 \\
K^{*}=L^{*}=600.032, \text { if } \lambda=1 \\
K^{*}=L^{*}=706.324, \text { if } \lambda=2
\end{array}\right.
$$

Note that, the competitive firm is just risk-averse when $\lambda=0$, while is regret-averse as well as risk-averse when $\lambda>0$.

From the findings of Case I, it's easy to verify $F_{K}\left(K^{*}, L^{*}\right)>\frac{r}{E p}, F_{L}\left(K^{*}, L^{*}\right)>\frac{w}{E p}$. In fact, we notice that the optimal capital and labor input under certain price, i.e., $p=\hat{p}=(4+8) / 2=6$, are $K^{* *}=L^{* *}=1296$, which are much larger than those under uncertain price. Therefore, the results we get from the illustration is in accordance with Theorem 1. Simultaneously, we get that the optimal input for the competitive firm with regret aversion under uncertainty are changing with the variation of $\lambda$ that is the weight of regret aversion relative to risk aversion. In this example, the optimal capital input and labor input are increasing with $\lambda$, put differently, that is $\partial K^{*} / \partial \lambda, \partial L^{*} / \partial \lambda>0$, which are in line with our result suggested in Theorem 2 because it can be shown that $\left(\hat{p} F_{K}-r\right) / F_{K}<\operatorname{Cov}\left(v^{\prime}(\pi),-p\right) / E v^{\prime}(\pi),\left(\hat{p} F_{L}-w\right) / F_{L}<\operatorname{Cov}\left(v^{\prime}(\pi),-p\right) / E v^{\prime}(\pi)$. In addition, by using $K^{*}=L^{*}$, we can plot the expected utility of profits, and the optimal input for $\lambda$ (See Figures 1-2 corresponding to Case I at length). Within them, Figure 1 confirms that for $\lambda=1, E u(\pi)$ achieves to the maximum at $K^{*}=600.032=L^{*}$. Figure 2. shows that the optimal input are increasing with $\lambda$. Of course, we can also plot the expected utility of profits, $E u(\pi)$, for other values of $\lambda$, but to avoid giving unnecessary details, we omit them in this paper.

Case I. The expected probability of $p=4$ is $q=1 / 3$.

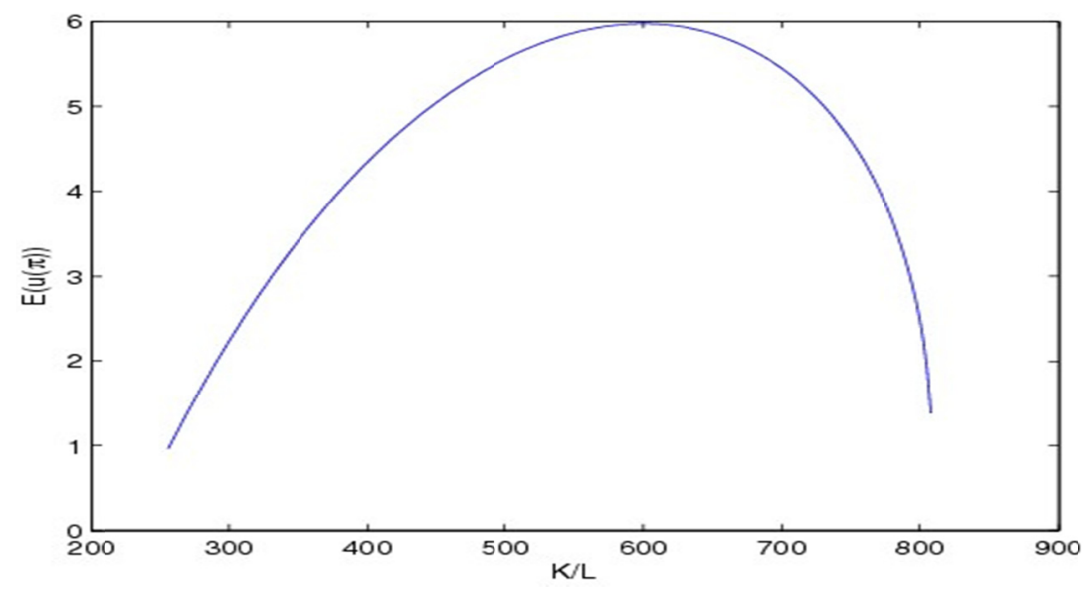

Figure 1. Expected utility of profits for $\lambda=1$

Note: $\mathrm{K} / \mathrm{L}$ herein indicates $K$ or $L$ (Hereafter the same). 


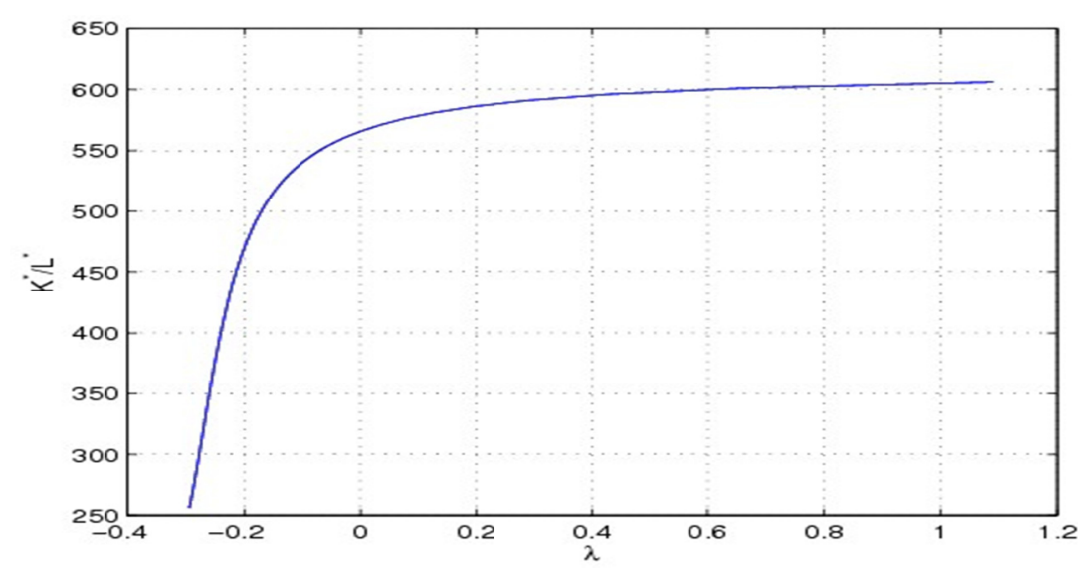

Figure 2. The dynamic relationships between optimal input and $\lambda$

In this example, $\partial K^{*} / \partial \lambda, \partial L^{*} / \partial \lambda>0$ imply that the optimal capital and labor input for the regret-aversecompetitive firm $(\lambda>0)$ under price uncertainty are larger than the optimal capital and labor input for the risk-averse competitive firm ( $\lambda=0$ ) under price uncertainty.

For different values of the expected probability $q$, we have the following consequences.

Case II. The expected probability of $p=4$ is $q=1 / 3$.

$$
\left\{\begin{array}{l}
K^{*}=L^{*}=688.538, \text { if } \lambda=0 \\
K^{*}=L^{*}=696.102, \text { if } \lambda=0.25 \\
K^{*}=L^{*}=699.805, \text { if } \lambda=0.5 \\
K^{*}=L^{*}=702.004, \text { if } \lambda=0.75 \\
K^{*}=L^{*}=703.454, \text { if } \lambda=1 \\
K^{*}=L^{*}=770.375, \text { if } \lambda=2
\end{array}\right.
$$

Case III. The expected probability of $p=4$ is $q=1 / 4$.

$$
\left\{\begin{array}{l}
K^{*}=L^{*}=741.054, \text { if } \lambda=0 \\
K^{*}=L^{*}=744.390, \text { if } \lambda=0.25 \\
K^{*}=L^{*}=746.052, \text { if } \lambda=0.5 \\
K^{*}=L^{*}=747.042, \text { if } \lambda=0.75 \\
K^{*}=L^{*}=747.699, \text { if } \lambda=1 \\
K^{*}=L^{*}=789.603, \text { if } \lambda=2
\end{array}\right.
$$

Analogously, corresponding to Case II and Case III, we can plot the expected utility of profits for $\lambda=1$ (See Figures 3-4. associated with Case II and Case III in detail).

Case II. The expected probability of $p=4$ is $q=1 / 3$. 


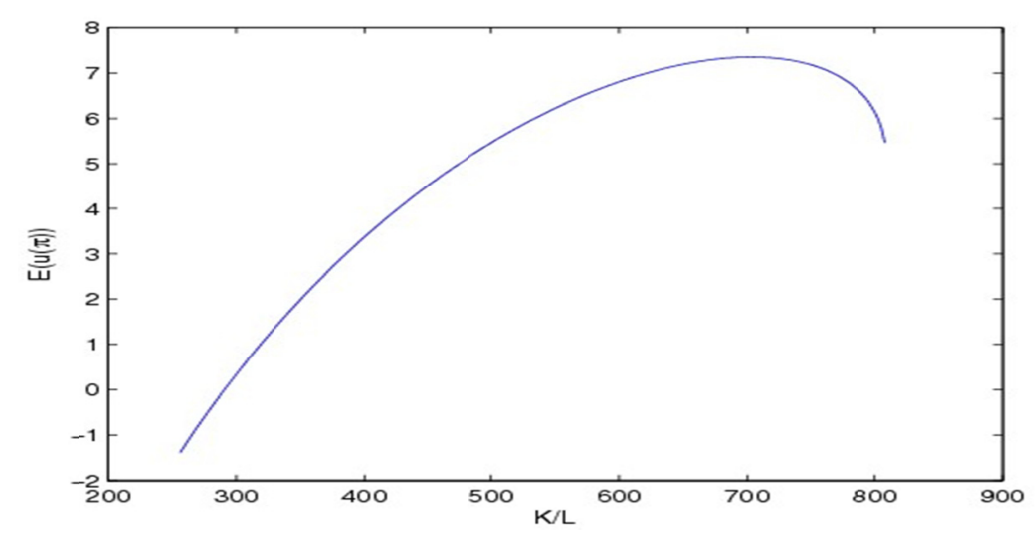

Figure 3. Expected utility of profits for $\lambda=1$

Case III. The expected probability of $p=4$ is $q=1 / 4$

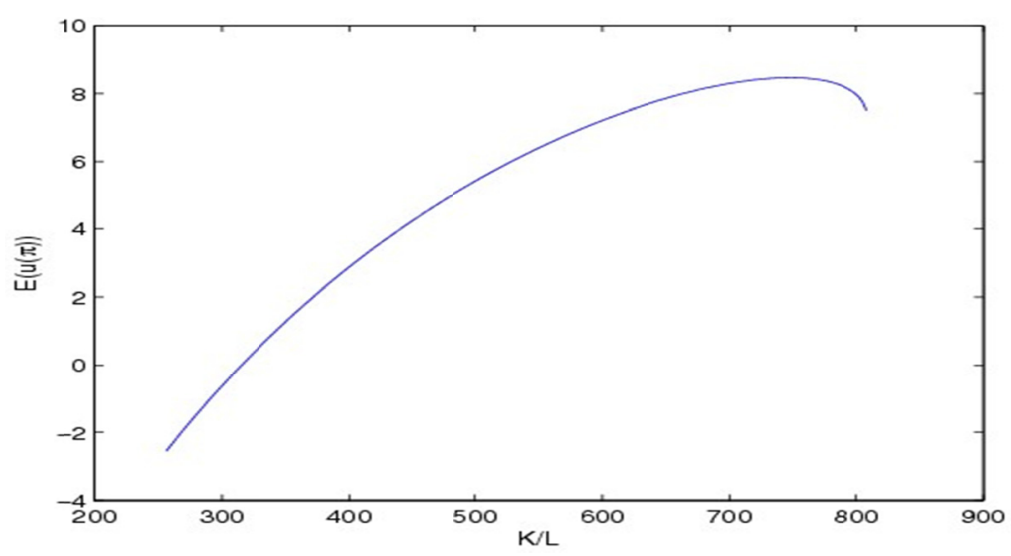

Figure 4. Expected utility of profits for $\lambda=1$

From the numerical results of Case I to Case III, we see that for the same $\lambda$, the optimal capital and labor input for the regret-averse competitive firm under price uncertainty are increasing with the decreasing of the expected probability of the bad case (The output price turns out to be low and sales turn out to be poor). Certainly, the expected utility of profits are increasing either. For example, given $\lambda=1$, we can see the $E u(\pi)$ in Figure 1 . $(\mathrm{q}=1 / 2)$ is the lowest, and $E u(\pi)$ in Figure $4(\mathrm{q}=1 / 4)$ is the highest, while $E u(\pi)$ in Figure 3. $(q=1 / 3)$ is between these two extremes. The consequence is not difficult to understand, because the lower the expected probability of the bad case, then the higher the expected probability of the good case (The output price turns out to be high and sales turn out to be good), which means the more positive the attitude of decision-maker toward the future, thereby, it's reasonable for the decision-maker to invest more.

Again, from the numerical results of Case I to Case III, we see that for the same scope of $\lambda$ such as $\lambda \in[0,2]$, the changes of optimal capital and labor input are more smoothing with the decreasing of the expected probability of the bad case $(p=4)$. In other words, the higher the expected probability of the good case $(p=8)$, the less impacts of the changes of $\lambda$, that is, the weight of regret-averse attribute relative to risk-averse attribute, would on the optimal capital and labor input.

\section{Conclusion}

During the past decades, many papers such as Kahneman and Tversky (1979) pointed out the conventional expected utility theory is invalid or non-universal in the real world. In other words, some important factors that can affect people's choices have been overlooked or misspecified by the conventional expected utility theory. In view of this point, Paroush and Venezia improved the traditional production theory by introducing a regret aversion factor. There exists, however, some limitations in the regret function they constructed.

To circumvent those limitations in Paroush and Venezia's paper, a more specific and tractable regret-averse 
function that can consider feelings of both regret and rejoicing at the same time is set up to study our problem in this paper. Fortunately, many results that are consistent with the behavior of regret-averse managers are obtained from the model we established in the paper. For instance, both the optimal capital and labor input of a competitive firm with regret aversion under price uncertainty are small relative to those of a competitive firm under certain price. Also, we show that the optimal input will change with the movement of the weight of regret aversion relative to risk aversion. Furthermore, the lower the expected probability of the bad case (The output price turns out to be low and sales turn out to be poor), then the higher the expected probability of the good case (The output price turns out to be high and sales turn out to be good), which means the more positive the attitude of decision-maker toward the future, thereby, the more investment would made by the decision-maker. Lastly, the higher the expected probability of the good case, the less impacts of the changes of the regret aversion weight relative to risk aversion would on the optimal capital and labor input.

In short, to assume that a firm is only risk-averse may not always be correct or appropriate though it is common in economic theory. That is why we extend the traditional production theory by introducing a regret aversion factor to a competitive firm under uncertainty. Put differently, we assume that a decision maker of a competitive firm under uncertain output price is not only risk-averse, but also regret-averse. Furthermore, our assumptions are consistent with some cases in the reality, which means our extension is sensible and reasonable, instead of taking it for granted.

The last but not the least, the major contribution of this paper can be concluded as below. First of all, this paper is the first to apply the two-attribute utility function to study the properties of a competitive firm's investment behavior with regret aversion and risk aversion under price uncertainty. Secondly, the model enables different firms to possess different regrets provided that their utility functions on the regret term are different, which circumvents the limitation that utility functions of regret-averse firms with different regret terms possesses the same regret. Finally, the framework allows us to make comparative statics of the optimal input by changing the parameter of the regret term. Certainly, the model not only represents the production theory for competitive firms with risk aversion, but also represents the production theory for competitive firms with regret aversion.

\section{Acknowledgements}

The author would like to express his sincere thanks to the anonymous referees for their helpful comments and suggestions, which greatly improved the presentation of this paper. The author also would like to thank the editors for their help.

This work is supported by Research Innovation Foundation of Shanghai University of Finance and Economics under Grant No. CXJJ-2011-336.

\section{References}

Bell, D. (1983). Regret in decison making under uncertainty. Operations Research, 30(5), 961-981. http://dx.doi.org/10.1287/opre.30.5.961

Braun, M., \& Muermann, A. (2004). The impact of regret on the demand for insurance. Journal of Risk and Insurance, 71(4), 737-767. http://dx.doi.org/10.1111/j.0022-4367.2004.00110.x

Hayashi, T. (2009). Stopping with anticipated regret. Journal of Mathematical Economics, 45(3), 479-490. http://dx.doi.org/10.1016/j.jmateco.2009.03.011

Kahneman, D., \& Tversky, A. (1979). Prospect theory: An analysis of decision under risk. Econometrica, 47(2), 263-292. http://dx.doi.org/10.2307/1914185

Laciana, C., \& Weber, E. (2008). Correcting expected utility for comparison between alternative outcomes: A unified parameterization of regret and disappointment. Journal of Risk and Insurance, 36(1), 1-17.

Landsberger, M., \& Subotnik, A. (1976). Optimal behavior of a monopolist facing a bicriteria objective function. International Economic Review, 17(3), 581-600. http://dx.doi.org/10.2307/2525790

Leland, H. (1972). Theory of the firm facing uncertain demand. American Economic Review, 62(3), 278-291. Retrieved from http://www.jstor.org/stable/1803376

Loomes, G., \& Sugden, R. (1982). Regret theory and alternative theory of rational choice under uncertainty. Economic Journal, 92(368), 805-824. http://dx.doi.org/10.2307/2232669

Ma, L., \& Xu, X. (2009). Regret aversion, two-fund separation and optimal assets allocation model. International Conference on Management and Service Scienc, 1-4. Retrieved from http://10.1109/ICMSS.2009.5301717 
Marschak, J. (1974). Economoic information, decision and prediction (III). Boston: D. Reidel Publicing Company.

Michenaud, S., \& Solink, B. (2008). Applying regret theory to investment choices: Currency hedging decisions. Journal of International Money and Finance, 27(3), 677-694. http://dx.doi.org/10.1016/j.jimonfin.2008.03.001

Muermann, A., Mitchell, O., \& Volkman, J. (2006). Regret, portfolio choice and guarantees in defined contribution schemes. Insurance, Mathematics and Economics, 39(2), 219-229. http://dx.doi.org/10.1016/j.insmatheco.2006.02.006

Mulandzi, M., Petersen, M., \& Schoeman, I. (2008). Optimal allocation between bank loans and treasuries with regret. Optimization Letters, 2(2), 555-566. http://dx.doi.org/10.1007/s11590-008-0082-9

Paroush, J., \& Venezia, I. (1979). On the theory of the competitive firm with a utility function defined on profits and regret. European Economic Review, 12(1), 193-202. http://dx.doi.org/10.1016/0014-2921(79)90001-1

Petersen, M. A., Mukuddem-Petersen, J., Mulaudzi, M. P., De Waal, B., \& Schoeman, I. M. (2010). Subprime risk and insurance with regret. Discrete Dynamics in Nature and Society, 4(1), 1-52. http://dx.doi.org/10.1155/2010/950413

Sandmo, A. (1971). On the theory of the competitive firm under price uncertainty. American Economic Review, 61(1), 65-73.

Renou, L., \& Schlag, K. H. (2010). Minimax regret and strategic uncertainty. Journal of Economic Theory, 145(1), 264-286. http://dx.doi.org/10.1016/j.jet.2009.07.005

Sheng, J. (2012). Behavioral asset pricing model based on regret theory. Fifth International Conference on Business Intelligence and Financial Engineering, 135-139. http://dx.doi.org/10.1007/s10994-005-0465-4

Stoltz, G., \& Lugosi, G. (2005). Internal regret in on-line portfolio selection. Machine Learning, 59(1), 125-159. http://dx.doi.org/10.1016/j.jet.2011.10.004

Stoye, J. (2011). Axioms for minimax regret choice correspondences. Journal of Economic Theory, 146(6), 2226-2251. http://doi:10.1016/j.jet.2011.10.004

Sugden, R. (1993). An axiomatic foundation of regret. Journal of Economic Theory, 60(1), 159-180. http://dx.doi.org/10.1006/jeth.1993.1039

Tsai, J. Y. (2012). Risk and regret aversions on optimal bank interest margin under capital regulation. Economic Modelling, 29(6), 2190-2197. http://dx.doi.org/10.1016/j.econmod.2012.06.028

Wong, K. (2011). Regret theory and the banking firm: The optimal bank interest margin. Economic Modelling, 28(7), 2483-2487. http://dx.doi.org/10.1016/j.econmod.2011.07.007

Wong, K. (2012). Production and insurance under regret aversion. Economic Modelling, 29(4), 1154-1160. http://dx.doi.org/10.1016/j.econmod.2012.04.001

\section{Copyrights}

Copyright for this article is retained by the author(s), with first publication rights granted to the journal.

This is an open-access article distributed under the terms and conditions of the Creative Commons Attribution license (http://creativecommons.org/licenses/by/3.0/). 